\title{
Photonic tuning of Beliaev damping in a superfluid
}

\author{
G. Kónya, G. Szirmai, D. Nagy, and P. Domokos \\ Institute for Solid State Physics and Optics, Wigner Research Centre, Hungarian Academy of Sciences, \\ P. O. Box 49, H-1525 Budapest, Hungary \\ (Received 29 December 2013; published 8 May 2014)
}

\begin{abstract}
We show that the Beliaev damping of elementary excitations in a homogeneous Bose-Einstein condensate can undergo resonant enhancement by several orders of magnitude when the superfluid is interacting with a far-detuned radiation field of an optical resonator. The photonic tuning of the quasiparticle damping can be controlled by an external laser drive.
\end{abstract}

Ultracold atoms coupled to the radiation field of an optical resonator form a long-range interacting many-body system [1-4] that proved to be suitable for the quantum simulation of the superradiant quantum phase transition of the Dicke model [5-7]. Critical behavior in nonequilibrium phase transitions between stationary phases of an open system [8-16] cannot be cast in the usual formalism of the symmetry-breaking transition of the ground state. As it has been predicted $[8,9]$ and recent experiments have proved [17], photon dissipation and the accompanying quantum fluctuations substantially modify the correlation functions and the critical exponents $[10,18]$. Dissipation is thus a key factor in quantum criticality. In addition, measured data revealed the effect of another dissipation channel related to atomic collisions [17]. In this paper we show that the damping rate of the soft mode can undergo an unexpected, resonancelike, huge enhancement prior to vanishing. The variation of the soft-mode frequency as the control parameter is tuned and the bath composed of the usually neglected phonon degrees of freedom is sampled at different points. At a certain value of the control parameter the spectral mode density diverges and the damping rate gets enhanced.

Elementary excitations of a homogeneous Bose-Einstein condensate of ultracold atoms are collective density waves with different wave numbers that can be considered quasiparticles. Besides the dispersion relation, the quasiparticles are characterized by a damping rate [19-24]. The finite lifetime originates from two possible scattering processes among quasiparticles. The first one leads to Landau damping [25-27], where the selected excitation, together with another thermally excited one, merges into a third excitation of the system. This mechanism needs a thermal occupation of the other excitation, therefore it vanishes at zero temperature. In the second, so-called Beliaev damping process [28,29], the selected excitation decays directly into two lower-energy excitations. This scattering process is the basic source of dissipation in a superfluid near zero temperature [30]. In the following, we will consider the Landau and Beliaev collisional decay processes of quasiparticles when the homogeneous Bose gas is in an optical resonator.

Suppose that a Bose-Einstein condensate of atoms is placed into an optical resonator [31] and is illuminated by a coherent laser light from the side perpendicular to the cavity axis (see Fig. 1). The laser frequency $\omega_{L}$ is far detuned from all atomic transitions and the absorption is thus negligibly small. However, the driving laser is close to resonance with a single mode of the cavity, hence the atoms can efficiently scatter laser light into this mode. The photon scattering between the driving laser and the cavity is subject to interference in the many-particle system. The cavity mode function selects density waves that are coupled by the collective scattering to the light field. Then the corresponding quasiparticles are sensitive to external control exerted by tuning the laser pump power or frequency.

At low temperature, the dilute gas of bosonic atoms with mass $m$, placed into a container with infinite length, is described by the Hamiltonian $(\hbar=1)$

$$
H_{A}=\int \hat{\Psi}^{\dagger}(x)\left[-\frac{1}{2 m} \frac{d^{2}}{d x^{2}}+\frac{g}{2} \hat{\Psi}^{\dagger}(x) \hat{\Psi}(x)\right] \hat{\Psi}(x) d x,
$$

where $g=4 \pi a / m w$ is the strength of the low-energy collisions with $s$-wave scattering length $a$. For simplicity we consider only the one-dimensional motion of the atoms along the cavity axis, inside an elongated trap with the transverse size of the condensate taken to be $w$. The entire Hamiltonian is then $H=H_{A}+H_{C}$, with

$$
\begin{aligned}
H_{C}= & -\Delta_{C} \hat{a}^{\dagger} \hat{a}+\int_{0}^{L} \hat{\Psi}^{\dagger}(x)\left[\eta\left(\hat{a}^{\dagger}+\hat{a}\right) \cos (k x)\right. \\
& \left.+U_{0} \hat{a}^{\dagger} \hat{a} \cos ^{2}(k x)\right] \hat{\Psi}(x) d x .
\end{aligned}
$$

The first term describes the bare cavity energy in a frame rotating with the pumping laser frequency $\Delta_{C}=\omega_{L}-\omega_{C}$. Terms in the integral represent two kinds of optical processes involving the atom cloud density. The first one is a laser drive of the cavity via photon scattering off the atoms. The corresponding effective amplitude is $\eta=\Omega g_{0} / \Delta_{A}$, with $\Omega$ the Rabi frequency of the pumping laser, $g_{0}$ the single-photon Rabi frequency in the cavity, and $\Delta_{A}=\omega_{L}-\omega_{A}$ the detuning of the laser from the atomic resonance. The cavity mode function is $\cos (k x)$, with wave number $k$. The second interaction term having $a^{\dagger} a$ is the absorption of cavity photons and induced emission back into the cavity [33-35]. This coherent scattering forms an optical lattice potential $\cos ^{2}(k x)$ for the atomic matter wave with a depth proportional to the intensity with a coefficient $U_{0}=g_{0}^{2} / \Delta_{A}$.

We briefly recall here that the system defined by this Hamiltonian admits a very simple solution for weak driving strength, which is referred to as the normal phase. When the density of the atom cloud is constant along the cavity axis, the effective driving term vanishes by integrating out the $\cos (k x)$ mode function over the condensate. This means destructive 


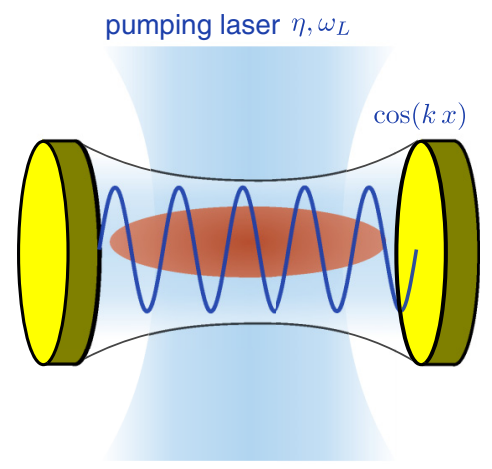

FIG. 1. (Color online) Scheme of the Fabry-Pérot resonator sustaining an electromagnetic standing wave with a single $\cos (k x)$ mode function and containing a Bose-Einstein condensate of atoms illuminated from the side.

interference in the scattering and no photon field builds up in the cavity. Nothing modulates then the quasihomogeneous condensate density. This solution breaks down above a certain pump power $\eta_{c}=\sqrt{-\Delta_{C} \omega_{R}}[5,6,36]$, where $\omega_{R}=k^{2} / 2 m$ is the recoil frequency. Above the critical point $\eta>\eta_{c}$ a stable periodic modulation of the atomic density is formed. However, in the following we consider only below-threshold driving strength with the corresponding homogeneous superfluid state.

The periodicity of the interaction terms with the cavity wavelength suggests the decomposition of the matter wave field in terms of Bloch states as

$$
\hat{\Psi}(x)=\frac{1}{\sqrt{L}} \sum_{q} e^{i q x}\left[\hat{b}_{q}+\sqrt{2} \cos (k x) \hat{c}_{q}+\sqrt{2} \sin (k x) \hat{s}_{q}\right],
$$

where $\hat{b}_{q}, \hat{c}_{q}$, and $\hat{s}_{q}$ are annihilation operators of atomic single-particle states with wave functions $e^{i q x}, \cos (k x) e^{i q x}$, and $\sin (k x) e^{i q x}$, respectively. Here we introduced a quasimomentum $q \in[-k / 2, k / 2]$. Note that states created by $\hat{c}_{q}$ and $\hat{s}_{q}$ carry a momentum $k$ in addition to the quasimomentum. We consider only the three lowest-energy bands; higher-order harmonics of the type $\cos (n k x)(n=2,3, \ldots)$ are not relevant for the present study [37].

All the boson mode operators can be split into mean-field and fluctuation parts. In the present geometry, for weak driving only the homogeneous atom field mode, which contains the condensate, has nonvanishing mean $\hat{b}_{0} \rightarrow \sqrt{N_{c}}+\hat{b}_{0}$, where $N_{c}$ is the number of condensate atoms. All the other excitation modes as well as the photon field have zero mean amplitude. The Heisenberg equations of motion for the fluctuations can be cast into the form of a hierarchy of terms with different powers of the condensate atom number $\sqrt{N_{c}}$,

$$
\begin{aligned}
i \frac{d}{d t} v_{\mu}= & \sum_{\nu} F_{\mu \nu} v_{\nu}+\frac{1}{\sqrt{N_{c}}} \sum_{q} \sum_{\alpha, \beta} V_{\mu}^{\alpha \beta}\left[w_{\alpha}^{\dagger}(q) w_{\beta}(q)\right. \\
& \left.-\left\langle w_{\alpha}^{\dagger}(q) w_{\beta}(q)\right\rangle\right], \\
i \frac{d}{d t} w_{\mu}(q)= & \sum_{\nu} G_{\mu \nu}(q) w_{\nu}(q)+\frac{1}{\sqrt{N_{c}}} \sum_{\alpha, \beta} W_{\mu}^{\alpha \beta} v_{\alpha} w_{\beta}(q),
\end{aligned}
$$

where we used the compact vector notation

$$
\begin{aligned}
v & =\left(a, a^{\dagger}, b_{0}, b_{0}^{\dagger}, c_{0}, c_{0}^{\dagger}, s_{0}, s_{0}^{\dagger}\right)^{T}, \\
w(q) & =\left(b_{q}, b_{-q}^{\dagger}, c_{q}, c_{-q}^{\dagger}, s_{q}, s_{-q}^{\dagger}\right)^{T}
\end{aligned}
$$

for the $q=0$ and $q \neq 0$ modes, respectively. Orders with higher powers of $1 / \sqrt{N_{c}}$ are omitted.

The highest order describes a linear coupling between the modes, which corresponds to the Bogoliubov approach. Eigenmodes of the linear system define the quasiparticles. Up to this order, modes with different quasimomentum magnitude $|q|$ do not couple. Moreover, only the $q=0$ modes couple to the photon degree of freedom by the laser-induced interaction (2). Therefore, the set of modes $\left\{a, b_{0}, c_{0}, s_{0}\right\}$, gathered in $v$ in Eq. (5a), form polariton modes and has to be treated separately. Of special importance is the excitation mode $c_{0}$, which matches exactly the cavity mode function $\cos (k x)$ and hence can be populated directly from the homogeneous Bose-Einstein condensate (BEC) mode $b_{0}$ by scattering photons between the laser and the cavity mode. The $q \neq 0$ quasimomentum excitations form the familiar Bogoliubov spectrum of the homogeneous BEC, represented by the dispersion curves in Figs. 2(b) and 2(c), and are referred to as phonons in the following.

Beyond the standard Bogoliubov approximation, the next order accounts for the interactions between quasiparticles, in particular, the cross coupling between polaritons and phonons. ${ }^{1}$ In Eq. (4), one polariton mode is coupled to two phonons, which is in accordance with the scattering processes underlying the Landau and the Beliaev damping, as sketched in Fig. 2. Assuming large condensate size, the phonons are spectrally dense and form a dissipation bath for the quasiparticles, including the phonons themselves. The damping is thus an intrinsic property originating from the short-range $s$-wave scattering. The rate of damping of a given polariton mode can be calculated within the Markov approximation [38], which relies on the phonons spanning a broad frequency range compared to the decay rate.

Figure 3 shows the Landau and the Beliaev damping rates, separately, of the polariton, which is composed dominantly of the $c_{0}$ mode. In the considered geometry, this quasiparticle is the most susceptible to the external control parameter $\eta$, which can be varied either by the pump laser power or by its detuning $\Delta_{A}$ from the atomic resonance.

The damping rate starts from the value characteristic of this excitation in free space and then the Beliaev part develops a strong resonance peak at $\eta / \eta_{c} \approx 0.8$. Further increasing the control parameter, the damping rate falls abruptly.

The tunability of the damping of a quasiparticle is due to the dressing by cavity photons and the broad range of tunability is due to the criticality in the system. The frequency of one of the polariton modes [resulting from the diagonalization of $F_{\mu \nu}$ in Eq. (4a)] depends significantly on the interaction strength, as presented in Fig. 2(a), since this is the soft mode of the

\footnotetext{
${ }^{1}$ Polariton-polariton and phonon-phonon couplings at this order are neglected. The former is highly nonresonant and the latter will be considered phenomenologically in the form of a finite phonon lifetime.
} 

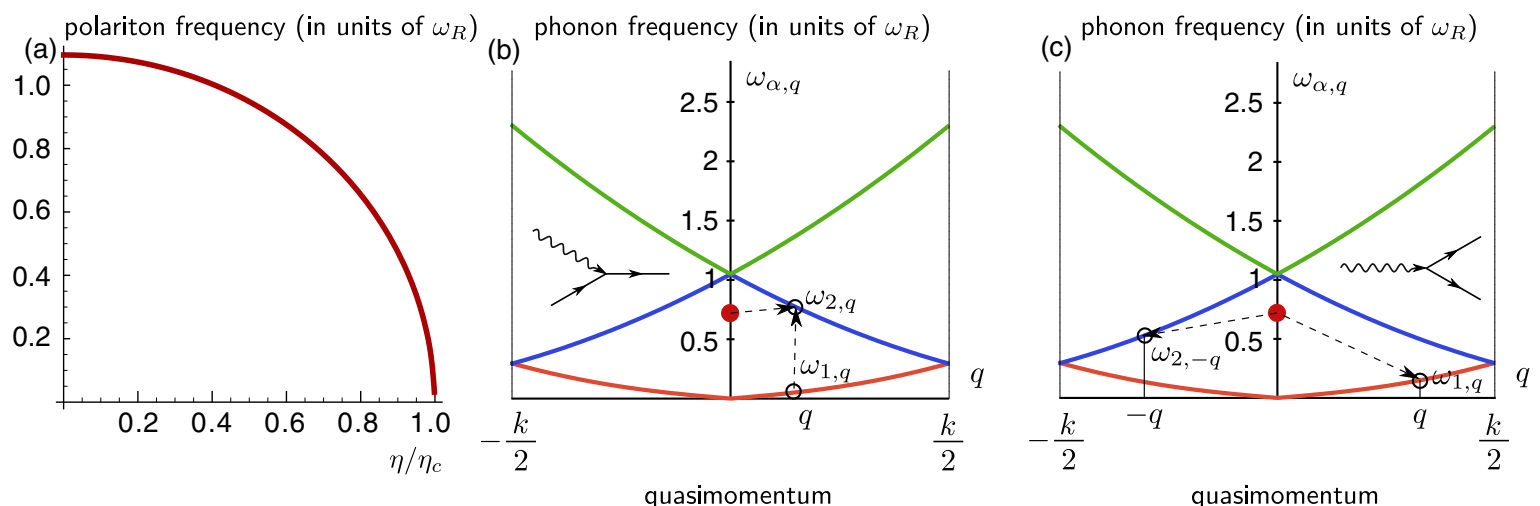

FIG. 2. (Color online) Frequency schemes. (a) Frequency of the relevant density-wave quasiparticle dressed by photons, the polariton mode, as a function of the laser power. This is the soft mode of the normal-superradiant phase transition [5,32], with vanishing frequency at the threshold of the superradiant phase. (b) and (c) Phonon dispersion relations as a function of the quasimomentum. (b) Illustration of Landau damping. The polariton together with a phonon decays into a higher-energy phonon. (c) Illustration of Beliaev damping. The polariton decays into two lower-energy phonons. In the damping processes energy and momentum are conserved.

normal-superradiant transition. It is important to note that this polariton is dominantly the $c_{0}$ density wave mode with a tiny admixture of photons, a maximum of $\omega_{R} /\left|\Delta_{C}\right|=10^{-3}$ for the parameters of the figure, due to the large eigenfrequency difference. For $\eta=0$ the polariton mode frequency is just at the point where the second and third phonon bands touch [see Figs. 2(b) and 2(c)]. When the laser is turned on, for increasing $\eta$ this particular point of the excitation branch departs from the dispersion curve and its frequency gradually decreases according to Fig. 2(a). For energy conservation, varying the polariton frequency amounts to sampling the bath at different points of the spectral density function. The resulting damping rates are thus tuned by the control parameter $\eta$.

The key to understanding the resonant behavior in the superfluid at zero temperature is that the spectral density function in this case is not directly the Bogoliubov phonon spectrum. In the normal phase, considered here, translation invariance and momentum conservation are preserved. In a Beliaev-type decay process, the $k$ momentum of the polariton is distributed between the two phonons. The only way is that the phonons have opposite quasimomenta and one of them has to be from the first and the other one from the second band, as illustrated schematically in Fig. 2(c). An effective spectral density function can be derived for such a third-order decay process. In particular, for polariton frequencies at about $\omega_{R} / 2$ the polariton decays into two phonons at the opposite edges of the Brillouin zone $|q| \approx k / 2$. Here the dispersion relation is linear, hence a continuum set of pairs $+q \lesssim k / 2$ on the lower branch and $-k / 2 \lesssim-q$ on the upper branch fulfills both the momentum and energy conservation laws. This yields a diverging effective spectral density and ultimately this is the underlying reason for the peak in the damping rate at $\eta / \eta_{c}=0.8$. Below this polariton frequency, the decay process becomes necessarily nonresonant and more and more suppressed. All this analysis is valid up to the point $\eta / \eta_{c}=1$, since this is a critical point where the homogeneous mean-field solution collapses.

The calculated polariton damping depends on the damping rates of the phonons that take part in the decay processes. The phonon linewidth could be derived from the microscopic model by taking into account the third-order phonon-phonon interaction terms, which we neglected in (4). The usual Landau and Beliaev formulas should be retained for the $q \neq 0$ phonon modes, as they do not couple to the photon field. Instead of this involved microscopic approach, we introduced the phenomenological parameter $\epsilon$, which accounts for the summed damping rates of the two phonons created by the polariton decay. By neglecting the dependence on the quasimomentum, we get a single, fitting parameter $\epsilon$. The polariton damping rate is displayed for various $\epsilon$ in the inset of Fig. 3. The phonon decay is typically in the range of a few hundreds hertz, therefore we expect the curve associated

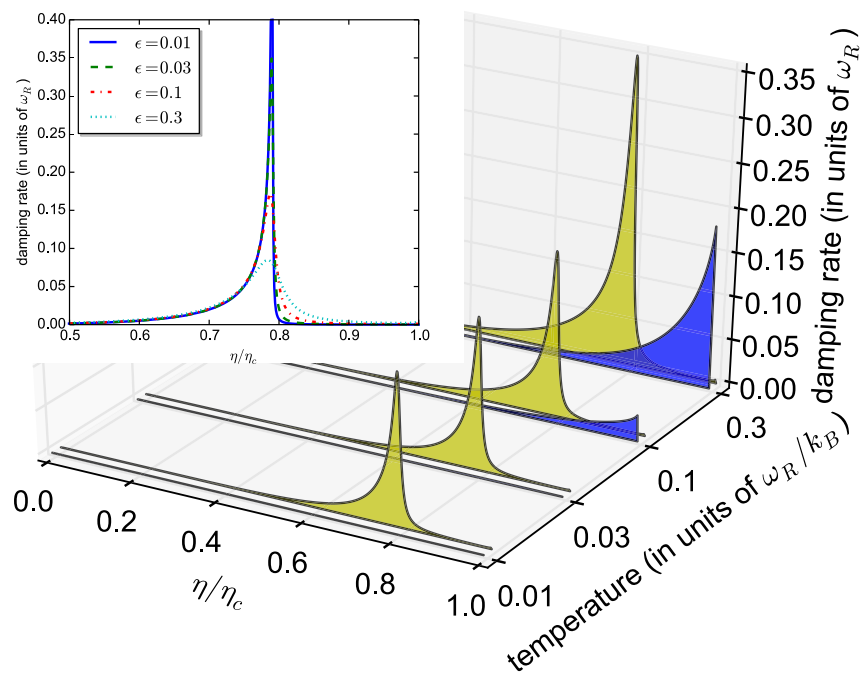

FIG. 3. (Color online) Landau (blue, front) and Beliaev (yellow, back) damping rates plotted as a function of the pumping strength $\eta$ at various temperatures. The Landau damping rate increases towards the critical point but is suppressed for temperatures below $k_{B} T \sim$ $0.1 \omega_{R}$. The inset shows the strong peak of the Beliaev damping (at $k_{B} T=0.01 \omega_{R}$, it hardly depends on temperature) for various values of the phenomenological parameter $\epsilon$ accounting for the decay rates of the phonons (in units of $\omega_{R}$ ). The parameters are $N_{c}=10^{4}$, $k L / 2 \pi=1000, N_{c} g / L=0.1 \omega_{R}$, and $\Delta_{C}=-1000 \omega_{R}$. 
with $\epsilon=0.1$ to be the best prediction of experimental data.

Recent experiments performed on this system found such a peak in the decay rate of the $\cos k x$ excitation mode (see Fig. 4 in [17]). Our theory reveals then that the observed peak can be a manifestation of the Beliaev damping, which is typically a negligible process, but here the light shift of the polariton frequency leads to a significant enhancement. The Beliaev damping relies on a nonlinear interaction of quasiparticles and on a continuum bath of phonons. Other effects such as the finite-size effect can also contribute [39] or modify the peak. In particular, the peak can be broadened by an external trapping, which might open a non-negligible gap in the dispersion relation at the edges of the Brillouin zone. We emphasize, however, that the photon-assisted Beliaev damping effect described above is an intrinsic property of the infinite ultracold-atom gas system. It exists in the thermodynamic limit defined by the length $L \rightarrow \infty$ and the number of atoms $N \rightarrow \infty$ such that the density $N / L$ is constant. Based on our detailed calculation [40], we expect that the damping rate is proportional to the density $N_{c} / L$. Our calculation was based on a one-dimensional model for the BEC, which captures properly the effect of resonant enhancement of the Beliaev damping. In a more accurate model including three-dimensional motion and eventually additional trapping potentials, the overlap integrals and thereby the coupling constants in Eqs. (4) would be modified by geometric factors. For the summation over the quasimomentum $q$ in the final expressions to be evaluated for the plots, we have taken the three-dimensional spectral density of phonon modes into account in order to suppress the contribution from the low-frequency part of the phonon bath.

We thank Ferdinand Brennecke, Rafael Mottl, and Peter Szépfalusy for discussions. This work was supported by the Hungarian National Office for Research and Technology under Contract No. ERC_HU_09 OPTOMECH, the Hungarian Academy of Sciences (Lendület Program No. LP2011-016), and the Hungarian Scientific Research Fund (Grant No. PD104652). G.S. also acknowledges support from the János Bolyai Scholarship.
[1] H. Ritsch, P. Domokos, F. Brennecke, and T. Esslinger, Rev. Mod. Phys. 85, 553 (2013).

[2] S. Gopalakrishnan, B. L. Lev, and P. M. Goldbart, Nat. Phys. 5, 845 (2009).

[3] P. Strack and S. Sachdev, Phys. Rev. Lett. 107, 277202 (2011).

[4] H. Jing, D. S. Goldbaum, L. Buchmann, and P. Meystre, Phys. Rev. Lett. 106, 223601 (2011).

[5] D. Nagy, G. Kónya, G. Szirmai, and P. Domokos, Phys. Rev. Lett. 104, 130401 (2010).

[6] K. Baumann, C. Guerlin, F. Brennecke, and T. Esslinger, Nature (London) 464, 1301 (2010).

[7] F. Piazza, P. Strack, and W. Zwerger, Ann. Phys. (NY) 339, 135 (2013).

[8] D. Nagy, G. Szirmai, and P. Domokos, Phys. Rev. A 84, 043637 (2011).

[9] B. Öztop, M. Bordyuh, Ö. E. Müstecaplığlu, and H. E. Türeci, New J. Phys. 14, 085011 (2012).

[10] E. G. Dalla Torre, S. Diehl, M. D. Lukin, S. Sachdev, and P. Strack, Phys. Rev. A 87, 023831 (2013).

[11] F. Dimer, B. Estienne, A. S. Parkins, and H. J. Carmichael, Phys. Rev. A 75, 013804 (2007).

[12] S. Morrison and A. S. Parkins, Phys. Rev. Lett. 100, 040403 (2008).

[13] S. Diehl, A. Tomadin, A. Micheli, R. Fazio, and P. Zoller, Phys. Rev. Lett. 105, 015702 (2010).

[14] B. Horstmann, J. I. Cirac, and G. Giedke, Phys. Rev. A 87, 012108 (2013).

[15] E. M. Kessler, G. Giedke, A. Imamoglu, S. F. Yelin, M. D. Lukin, and J. I. Cirac, Phys. Rev. A 86, 012116 (2012).

[16] N. Aggarwal, S. Mahajan, and A. B. Bhattacherjee, J. Mod. Opt. 60, 1263 (2013).

[17] F. Brennecke, R. Mottl, K. Baumann, R. Landig, T. Donner, and T. Esslinger, Proc. Natl. Acad. Sci. USA 110, 11763 (2013).

[18] E. G. Dalla Torre, E. Demler, T. Giamarchi, and E. Altman, Nat. Phys. 6, 806 (2010).

[19] D. S. Jin, M. R. Matthews, J. R. Ensher, C. E. Wieman, and E. A. Cornell, Phys. Rev. Lett. 78, 764 (1997).
[20] F. Chevy, V. Bretin, P. Rosenbusch, K. W. Madison, and J. Dalibard, Phys. Rev. Lett. 88, 250402 (2002).

[21] E. E. Rowen, N. Bar-Gill, R. Pugatch, and N. Davidson, Phys. Rev. A 77, 033602 (2008).

[22] W. V. Liu, Phys. Rev. Lett. 79, 4056 (1997).

[23] S. Giorgini, Phys. Rev. A 57, 2949 (1998).

[24] P. O. Fedichev, G. V. Shlyapnikov, and J. T. M. Walraven, Phys. Rev. Lett. 80, 2269 (1998).

[25] M. Guilleumas and L. P. Pitaevskii, Phys. Rev. A 61, 013602 (1999).

[26] J. Reidl, A. Csordás, R. Graham, and P. Szépfalusy, Phys. Rev. A 61, 043606 (2000).

[27] B. Jackson and E. Zaremba, New J. Phys. 5, 88 (2003).

[28] E. Hodby, O. M. Maragò, G. Hechenblaikner, and C. J. Foot, Phys. Rev. Lett. 86, 2196 (2001).

[29] N. Katz, J. Steinhauer, R. Ozeri, and N. Davidson, Phys. Rev. Lett. 89, 220401 (2002)

[30] Y. Kagan and L. A. Maksimov, Phys. Rev. A 64, 053610 (2001).

[31] F. Brennecke, T. Donner, S. Ritter, T. Bourdel, M. Köhl, and T. Esslinger, Nature (London) 450, 268 (2007).

[32] R. Mottl, F. Brennecke, K. Baumann, R. Landig, T. Donner, and T. Esslinger, Science 336, 1570 (2012).

[33] K. W. Murch, K. L. Moore, S. Gupta, and D. M. Stamper-Kurn, Nat. Phys. 4, 561 (2008).

[34] M. Wolke, J. Klinner, H. Kessler, and A. Hemmerich, Science 337, 75 (2012).

[35] D. Schmidt, H. Tomczyk, S. Slama, and C. Zimmermann, Phys. Rev. Lett. 112, 115302 (2014).

[36] K. Baumann, R. Mottl, F. Brennecke, and T. Esslinger, Phys. Rev. Lett. 107, 140402 (2011).

[37] G. Konya, G. Szirmai, and P. Domokos, Eur. Phys. J. D 65, 33 (2011).

[38] R. Graham, J. Stat. Phys. 101, 243 (2000).

[39] M. Kulkarni, B. Öztop, and H. E. Türeci, Phys. Rev. Lett. 111, 220408 (2013).

[40] G. Kónya, G. Szirmai, and P. Domokos (unpublished). 